\title{
UBE2D1 RNA Expression Was an Independent Unfavorable Prognostic Indicator in Lung Adenocarcinoma, but Not in Lung Squamous Cell Carcinoma
}

\author{
Liyan Hou, ${ }^{1}$ Yingbo Li, ${ }^{2}$ Ying Wang, ${ }^{3}$ Dongqiang $\mathrm{Xu},{ }^{3}$ Hailing Cui, ${ }^{3}$ Xiaoyan $\mathrm{Xu},{ }^{3}$ \\ Ying Cong, ${ }^{1}$ and Chengyong $\mathrm{Yu} \mathbb{1}^{3}$ \\ ${ }^{1}$ Department of Pharmacy, Weihai Central Hospital, Weihai, 264400 Shandong, China \\ ${ }^{2}$ Department of Blood Transfusion, Weihai Central Hospital, Weihai, 264400 Shandong, China \\ ${ }^{3}$ Clinical Laboratory, Weihai Central Hospital, Weihai, 264400 Shandong, China \\ Correspondence should be addressed to Chengyong Yu; chengyongyu@foxmail.com
}

Received 25 June 2018; Accepted 2 September 2018; Published 21 October 2018

Academic Editor: George Agrogiannis

Copyright (C) 2018 Liyan Hou et al. This is an open access article distributed under the Creative Commons Attribution License, which permits unrestricted use, distribution, and reproduction in any medium, provided the original work is properly cited.

\begin{abstract}
In this study, we investigated the potential prognostic value of ubiquitin-conjugating enzyme E2D1 (UBE2D1) RNA expression in different histological subtypes of non-small-cell lung cancer (NSCLC). A retrospective study was performed by using molecular, clinicopathological, and survival data in the Cancer Genome Atlas (TCGA)-Lung Cancer. Results showed that both lung adenocarcinoma (LUAD) $(N=514)$ and lung squamous cell carcinoma (LUSC) $(N=502)$ tissues had significantly elevated UBE2D1 RNA expression compared to the normal tissues ( $p<0.001$ and $p=0.036$, respectively). UBE2D1 RNA expression was significantly higher in LUAD than in LUSC tissues. Increased UBE2D1 RNA expression was independently associated with shorter OS (HR: 1.359, 95\% CI: 1.031-1.791, $p=0.029$ ) and RFS (HR: $1.842,95 \%$ CI: $1.353-2.508, p<0.001$ ) in LUAD patients, but not in LUSC patients. DNA amplification was common in LUAD patients $(88 / 551,16.0 \%)$ and was associated with significantly upregulated UBE2D1 RNA expression. Based on these findings, we infer that UBE2D1 RNA expression might only serve as an independent prognostic indicator of unfavorable OS and RFS in LUAD, but not in LUSC.
\end{abstract}

\section{Introduction}

Ubiquitination is a biological process, in which the targeting proteins were modified with ubiquitin for degradation [1]. This process is critical for cellular homeostasis, DNA repair, and proteasomal degradation [1]. Ubiquitination involves at least three classes of enzymes, including ubiquitin-activating enzymes (E1s), ubiquitin-conjugating enzymes (E2s), and ubiquitin-protein ligases (E3s) [1, 2]. E2s mediate ubiquitination by selective interactions with E1s and E3s and are responsible for the E3 selection and substrate modification, thus playing a critical role in ubiquitin transfer [3, 4]. They dictate the synthesis of a mono- or polyubiquitinated chain of a specific lysine linkage, which subsequently determines the fate of the substrate: proteasomal degradation or signaling $[3,4]$.
The ubiquitin-conjugating enzyme E2D family constitutes three members, including UBE2D1, UBE2D2, and UBE2D3, which all belong to the E2s. These family members share over $88 \%$ sequence identity and thus have similar enzymic activity $[5,6]$. Previous studies found that UBE2D dysregulation is involved in some important pathways in carcinogenesis. They mediate the ubiquitination of the tumor-suppressor protein p53 [7-9]. Suppression of UBE2Ds can stabilize p53, leading to enhanced apoptosis and markedly inhibited proliferation of human lung cancer cells in a p53-dependent manner [10]. Among the three members, UBE2D1 can collaborate with cellular inhibitor of apoptosis protein 1 (cIAP1) and mediate tumor necrosis factor $\alpha$ - (TNF $\alpha$-) stimulated receptor-interacting protein 1 (RIP1) ubiquitination and NF-kappaB activation [11]. One recent study found that Smad ubiquitination regulatory factor 2 (SMURF2) 


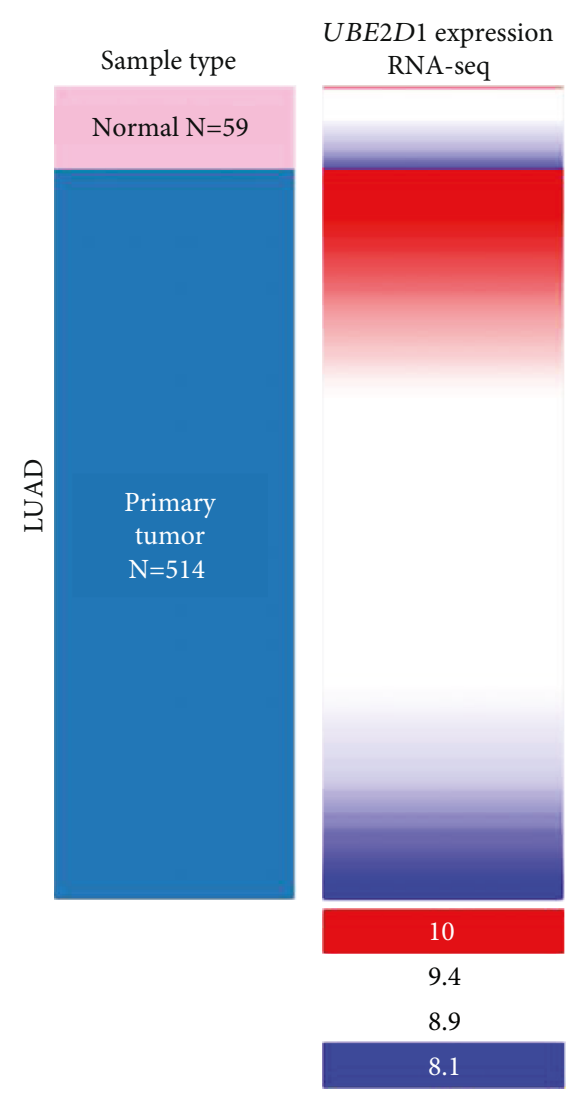

Log2 (norm_count+1)

(a)

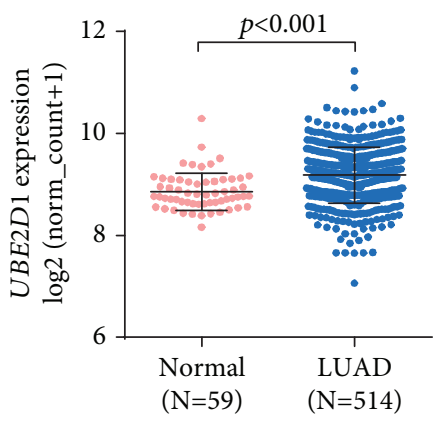

(c)

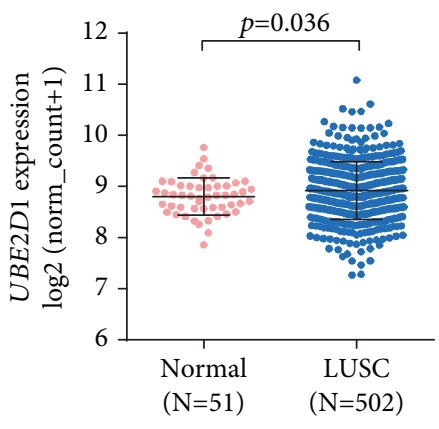

(d)

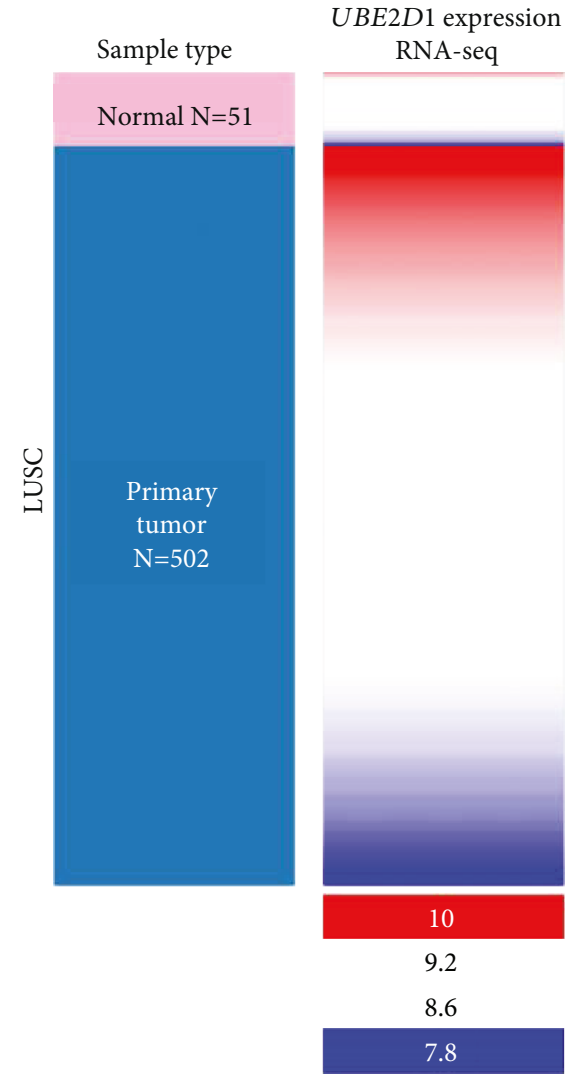

$\log 2$ (norm_count +1$)$

(b)

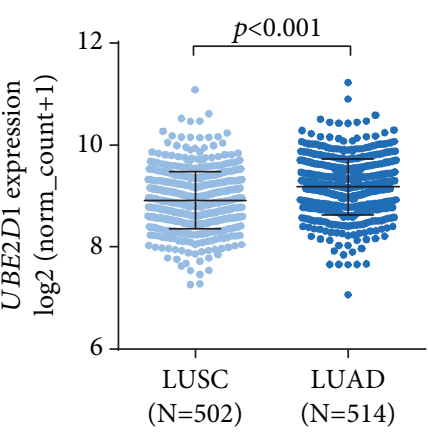

(e)

FIGURE 1: UBE2D1 RNA expression was upregulated in both LUAD and LUSC tissues compared to normal lung tissues (a-d). Heatmap (a-b) and plot chart $(\mathrm{b}-\mathrm{c})$ of UBE2D1 RNA expression in LUAD $(N=514)$ and LUSC $(N=502)$ tissues and their corresponding normal lung tissues. (e) Comparison of UBE2D1 RNA expression between LUAD and LUSC tissues.

and UBE2D1 form a critical E3:E2 complex, which maintains Kirsten Ras (KRAS) protein stability [12]. Interruption of this complex by siRNA reduces the clonogenic survival in vitro and increases tumor latency in vivo in cancer cells including mutant KRAS-driven tumors [12]. These findings suggest that UBE2D1 might regulate some critical cancerrelated signaling pathways.

In this study, by using the data from the Cancer Genome Atlas (TCGA)-Lung Cancer, we examined the expression profile of UBE2D1 in the two major subtypes of non-smallcell lung cancer (NSCLC) lung adenocarcinoma (LUAD) and lung squamous cell carcinoma (LUSC) and its prognostic value in these subtypes.

\section{Materials and Methods}

2.1. Retrospective Analysis Using the Data from the Cancer Genome Atlas (TCGA). This study is a retrospective study using the data from TCGA, with access provided by the UCSC Xena browser (https://xenabrowser.net/). In TCGA, molecular, clinicopathological, and over 10-year survival data of more than 500 LUAD or LUSC patients were recorded. Generally, in TCGA-LUAD, tumor tissues from 514 patients with primary tumors were collected for the RNA-seq study. 502 out of the 514 patients had intact OS data recorded. In TCGA-LUSC, tumor tissues from 501 patients with primary tumors were collected for the RNA- 


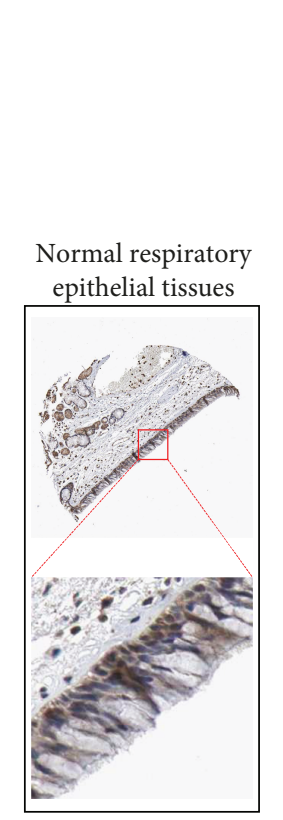

(a)

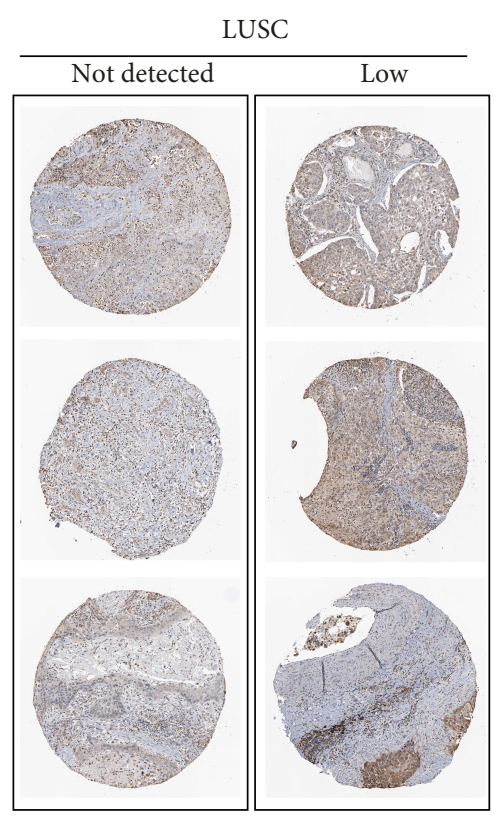

(b)

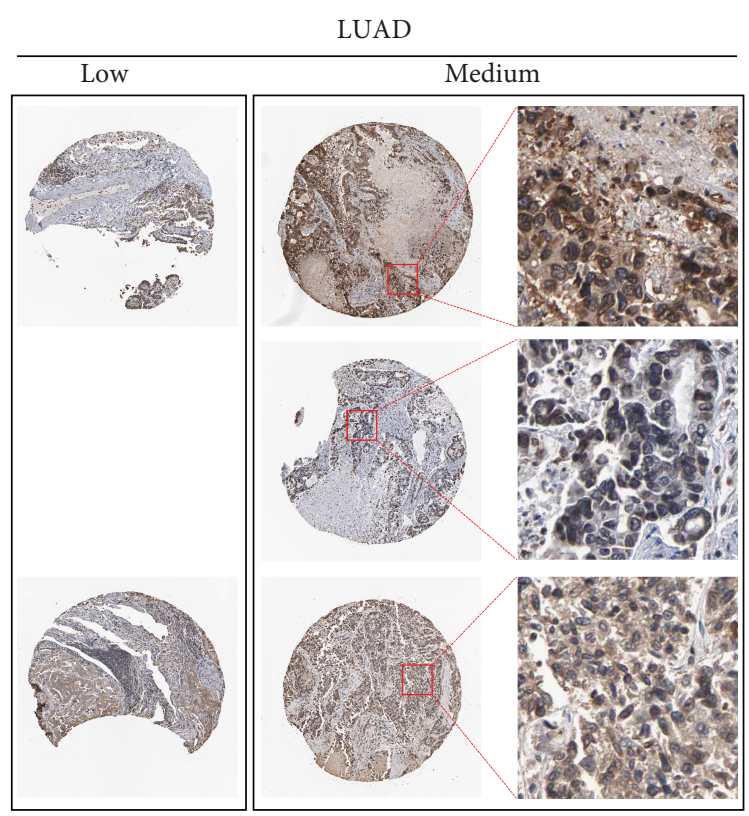

(c)

FIGURE 2: UBE2D1 protein expression in normal respiratory epithelium and LUAD and LUSC tissues. UBE2D1 IHC staining in normal respiratory epithelial tissues (a), in LUSC tissues (b), and in LUAD tissues (c).

seq study. 494 out of the 501 patients had intact OS data recorded. Clinicopathological parameters of patients with primary LUAD, including age at diagnosis, gender, smoking history, clinical stage, nodal invasion, residual tumors, recurrence status, RFS in days, living status, and OS in days, were downloaded for survival-related analysis. KaplanMeier curves of OS and RFS were generated to examine the survival difference in patients with high/low UBE2D1 RNA expression.

The genomic data, including UBE2D1 DNA copy number alterations (CNAs), which were presented as gene-level thresholded GISTIC2-processed copy number data, were also downloaded to examine the association between UBE2D1 RNA expression and the CNAs in LUAD patients.

2.2. Immunohistochemistry (IHC) Examination of UBE2D1 Protein Expression. UBE2D1 expression at the protein level in normal respiratory epithelial tissues and LUAD and LUSC tissues was characterized by using IHC staining data in the Human Protein Atlas (HPA) (https://www.proteinatlas. org/) $[13,14]$. IHC scoring in the database was performed by combining staining intensity (negative, low, moderate, or strong) and fraction of stained cells $(<25 \%, 25-75 \%$, or $>75 \%$ ) [15]. Each combination of intensity and fractions is automatically converted into a protein expression level score as follows: not detected: negative (-), weak: $<25 \%$, low: weak combined with either $25-75 \%$ or $75 \%$, moderate: $<25 \%$, medium: moderate combined with either $25-75 \%$ or $75 \%$, strong: $<25 \%$, and high: medium/strong combined with either $25-75 \%$ or $75 \%$ [15].

2.3. Statistical Analysis. Statistical analysis was conducted by using GraphPad Prism 6.0 (GraphPad Inc., La Jolla, CA,
USA) or SPSS 19.0 software package (SPSS Inc., Chicago, IL, USA). Welch's $t$-test was performed to examine the difference of UBE2D1 RNA expression. Receiver operating characteristic (ROC) analysis for death and recurrence detection was performed to identify the Youden index for UBE2D1 RNA expression (the cutoff to separate patients) in KaplanMeier curves of OS and RFS. The association between UBE2D1 RNA expression and the clinicopathological parameters in LUAD patients was assessed by using the chi-squared test by two-sided Fisher's exact test. The log-rank test was conducted to examine the significance of the difference between the curves. Univariate and multivariate Cox regression models were used to evaluate the prognostic significance of UBE2D1 RNA expression. $p<0.05$ was considered statistically significant.

\section{Results}

3.1. UBE2D1 RNA Expression Was Higher in LUAD and LUSC Tissues than in Normal Respiratory Tissues. By data mining in TCGA, we obtained the RNA-seq data of UBE2D1 expression in NSCLC tissues and in normal lung tissues. The comparison showed that both LUAD $(N=514)$ and LUSC $(N=502)$ tissues had significantly elevated UBE2D1 RNA expression compared to the normal tissues $(p<0.001$ and $p=0.036$, Figures $1(\mathrm{a})-1(\mathrm{~d})$ ). However, LUAD tissues had substantially higher expression of UBE2D1 RNA expression compared to LUSC tissues $(p<0.001$, Figure 1(e)). Using IHC staining data in the HPA, we also assessed UBE2D1 expression at the protein level in cancer tissues and normal lung tissues. Results showed that normal respiratory tissues usually had low UBE2D1 expression (Figure 2(a)). Among 6 cases of LUSC tissues examined, 3 cases had 


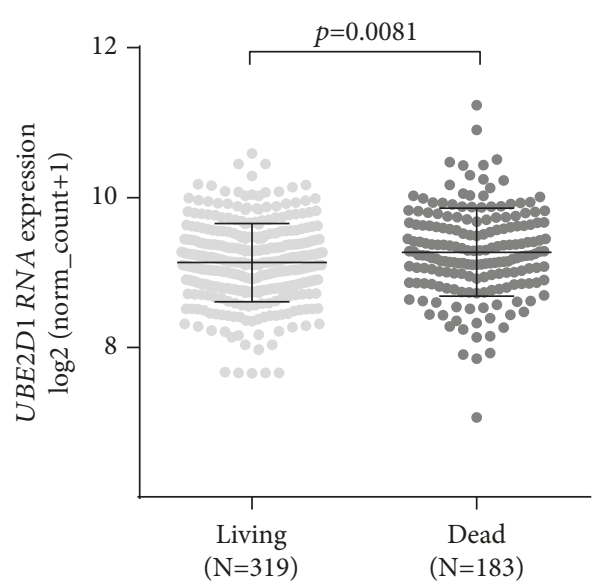

(a)

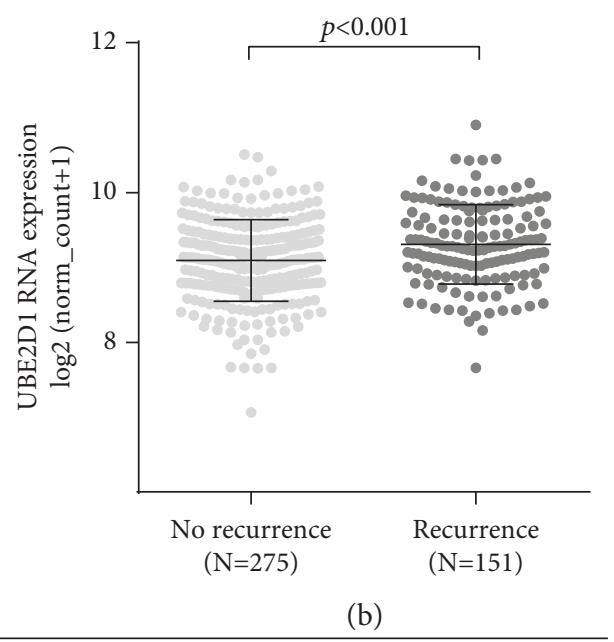

LUAD

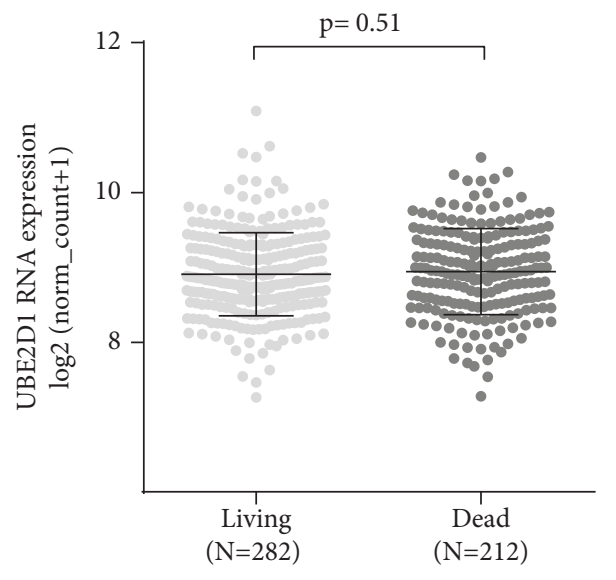

(c)

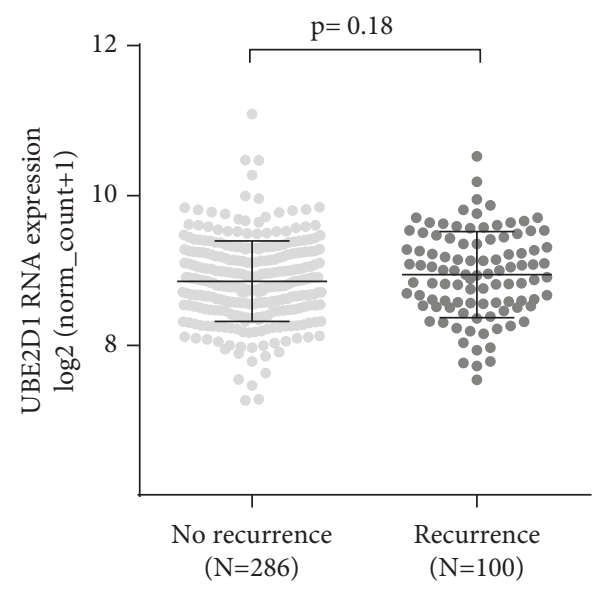

(d)

LUSC

FIgURE 3: Comparison of UBE2D1 RNA expression in LUAD/LUSC patients with different survival outcomes (a-d). Comparison of UBE2D1 RNA expression in LUAD (a-b) and LUSC $(c-d)$ patients according to their living status $(a, c)$ and recurrence status (b, d).

negative expression, while the rest 3 cases had low expression (Figure 2(b)). Among 5 cases of LUAD tissues examined, 2 cases had low expression and 3 cases had medium expression (Figure 2(c)). These findings suggest that UBE2D1 might also be upregulated at the protein level in LUAD tissues.

\subsection{Increased UBE2D1 RNA Expression Was Associated} with Poor Survival Outcomes in LUAD Patients, but Not in LUSC Patients. Then, we assessed the association between UBE2D1 RNA expression and survival outcomes in the major subtypes of NSCLC patients. The deceased LUAD cases $(N=183)$ had markedly higher UBE2D1 RNA expression compared to the living cases $(N=319)$ (Figure $3(\mathrm{a})$ ). Besides, the LUAD patients with recurrence $(N=151)$ also had significantly increased UBE2D1 RNA expression compared to the patients without recurrence $(N=275)$ (Figure 3(b)). In comparison, we did not find any significant associations in LUSC (Figures 3(c) and 3(d)). Then, we generated Kaplan-Meier survival curves to examine the association between UBE2D1 RNA expression and the survival outcomes. Results showed that in LUAD patients, the group with high UBE2D1 RNA expression had inferior OS $(p=0.0033)$ and RFS $(p=0.0011)$ compared to the group with low expression (Figures $4(\mathrm{a})$ and 4(b)). In contrast, UBE2D1 RNA expression was not associated with OS or RFS in LUSC patients $(p=0.93$ and 0.41 , respectively, Figures 4(c) and 4(d)).

3.3. UBE2D1 RNA Expression Was Independently Associated with Shorter OS and RFS in LUAD Patients. The association between UBE2D1 RNA expression and the clinicopathological parameters in $L U A D$ patients is summarized in Table 1. The comparison showed that the group with high UBE2D1 RNA expression had a significantly higher ratio of patients in advanced stages (III/IV) (34/119 vs. 72/375, $p=0.04)$, nodal positive cases (50/120 vs. $117 / 371, p=0.046)$, cases with residual tumors ( $8 / 91$ vs. $8 / 261, p=0.037$ ), recurrence (48/101 vs. $103 / 325, p=0.0043)$, and death (59/122 vs. $124 /$ $380, p=0.0024)$ compared to the group with low UBE2D1 RNA expression. 


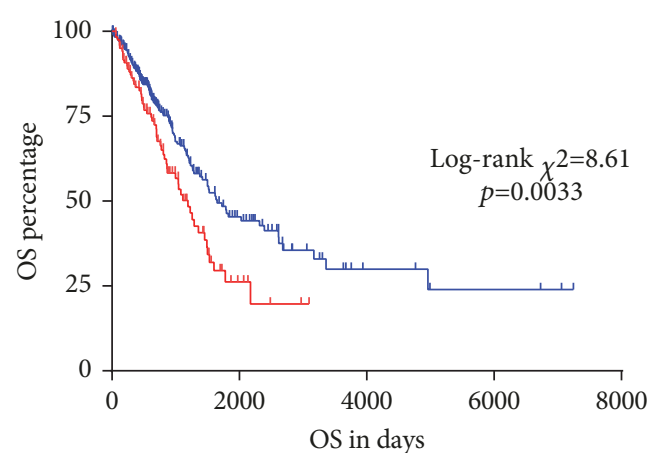

UBE2D1 RNA expression

+ High $(\mathrm{N}=122)$

$\perp \operatorname{Low}(\mathrm{N}=380)$

(a)

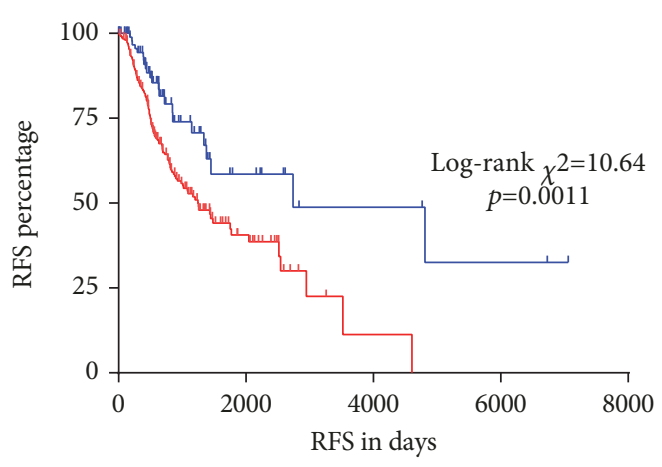

UBE2D1 RNA expression

$+\operatorname{High}(\mathrm{N}=326)$

$+\operatorname{Low}(\mathrm{N}=100)$

(b)

LUAD

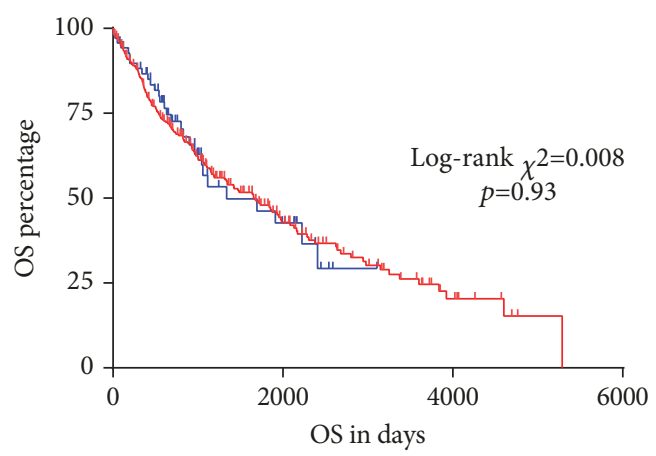

UBE2D1 RNA expression

$+\operatorname{High}(\mathrm{N}=424)$

$+\operatorname{Low}(\mathrm{N}=70)$

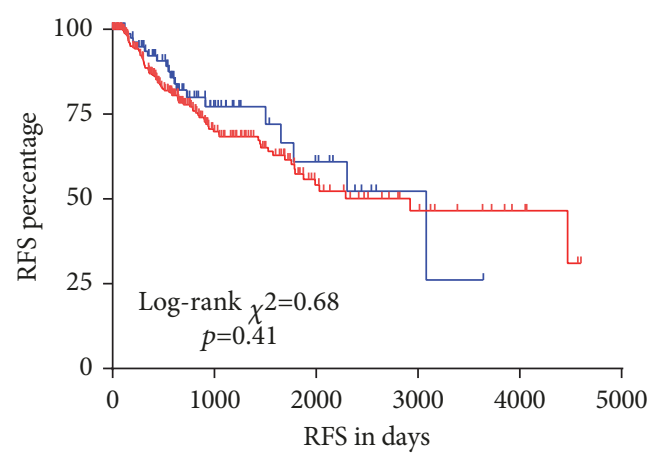

UBE2D1 RNA expression

$+\operatorname{High}(\mathrm{N}=298)$

$+\operatorname{Low}(\mathrm{N}=88)$

(c)

(d)

LUSC

FIGURE 4: Kaplan-Meier curves of OS and RFS in LUAD and LUSC (a-d). Kaplan-Meier curves of OS (a, c) and RFS (b, d) in LUAD $(\mathrm{a}-\mathrm{b})$ and LUSC (c-d) patients. The patients were grouped according to the Youden Index identified in ROC analysis for death and recurrence detection.

In univariate analysis, we observed that advanced stages, positive nodal invasion with residual tumors, and increased UBE2D1 RNA expression were associated with unfavorable OS and RFS in LUAD (Tables 2 and 3). The following multivariate analysis confirmed that increased UBE2D1 RNA expression independently predicted poor OS (HR: 1.359, 95\% CI: $1.031-1.791, p=0.029$ ) (Table 2) and RFS (HR: 1.842 , 95\% CI: $1.353-2.508, p<0.001$ ) (Table 3).

\subsection{DNA CNAs Were Associated with Dysregulated UBE2D1} RNA Expression in LUAD Patients. Then, we explored the potential mechanism of UBE2D1 dysregulation. Among 551 cases with both UBE2D1 CNAs and RNA-seq data, 88 cases had amplification $(+1 /+2)$ CNA, 295 had copy neutral (0) CNA, and 128 had deletion (-1/-2) CNA (Figure 5). DNA deletion was associated with decreased UBE2D1 RNA expression, while DNA amplification was associated with increased UBE2D1 RNA expression, compared to the copy neutral group (Figure 5).

\section{Discussion}

In this study, our data mining results showed that although UBE2D1 RNA was significantly upregulated in both LUAD and LUSC tissues compared with normal tissues, its expression was even higher in LUAD tissues than in LUSC tissues. Interestingly, we observed that UBE2D1 RNA upregulation was associated with poor survival outcomes in LUAD patients, but not in LUSC patients. By performing univariate and multivariate analyses, we confirmed that increased UBE2D1 RNA expression was independently associated with shorter OS (HR: 1.359, 95\% CI: 1.031-1.791, $p=0.029$ ) and RFS (HR: 1.842 , 95\% CI: 1.353-2.508, $p<0.001$ ) in LUAD patients. Therefore, we infer that UBE2D1 RNA expression might only serve as an independent prognostic indicator of unfavorable OS and RFS in LUAD, but not in LUSC.

Previous studies showed that UBE2Ds play a critical role in the ubiquitination and degradation of p53 [7, 8]. P53 is a master tumor-suppressive gene, and its degradation has a 
TABLE 1: The associations between UBE2D1 RNA expression and the clinicopathological parameters of patients with LUAD.

\begin{tabular}{|c|c|c|c|c|}
\hline \multirow{2}{*}{ Parameters } & & \multicolumn{2}{|c|}{ UBE2D1 RNA expression } & \multirow{2}{*}{$p$ value } \\
\hline & & $\operatorname{High}(N=122)$ & Low $(N=380)$ & \\
\hline Age (mean \pm SD) & & $64.54 \pm 10.99$ & $65.58 \pm 9.59$ & 0.91 \\
\hline \multirow{2}{*}{ Gender } & Female & 65 & 206 & \multirow{2}{*}{0.92} \\
\hline & Male & 57 & 174 & \\
\hline \multirow{3}{*}{ Smoking history } & $2 / 3 / 4 / 5$ & 108 & 308 & \multirow{3}{*}{0.054} \\
\hline & 1 & 11 & 61 & \\
\hline & No data & 3 & 11 & \\
\hline \multirow{3}{*}{ Clinical stage } & III/IV & 34 & 72 & \multirow{3}{*}{0.040} \\
\hline & $\mathrm{I} / \mathrm{II}$ & 85 & 303 & \\
\hline & Discrepancy/no data & 3 & 5 & \\
\hline \multirow{3}{*}{ Nodal invasion } & N0 & 70 & 254 & \multirow{3}{*}{0.046} \\
\hline & $\mathrm{N} 1 / 2 / 3$ & 50 & 117 & \\
\hline & $\mathrm{NX} /$ no data & 2 & 9 & \\
\hline \multirow{3}{*}{ Residual tumors } & R0 & 83 & 253 & \multirow{3}{*}{0.037} \\
\hline & $\mathrm{R} 1 / \mathrm{R} 2$ & 8 & 8 & \\
\hline & $\mathrm{RX} /$ no data & 31 & 119 & \\
\hline \multirow{3}{*}{ Recurrence status } & No & 53 & 222 & \multirow{3}{*}{0.0043} \\
\hline & Yes & 48 & 103 & \\
\hline & No data & 21 & 55 & \\
\hline \multirow{2}{*}{ Living status } & Living & 63 & 256 & \multirow{2}{*}{0.0024} \\
\hline & Dead & 59 & 124 & \\
\hline
\end{tabular}

Smoking history: 1-lifelong nonsmoker, 2-current smoker, 3-current reformed smoker (for $>15$ yrs), 4 -current reformed smoker (for $\leq 15$ yrs), and 5-current reformed smoker (duration not specified); NX: regional lymph nodes cannot be assessed; RX: the presence of residual tumor cannot be assessed.

TABLE 2: Univariate and multivariate analyses of overall survival in patients with LUAD.

\begin{tabular}{|c|c|c|c|c|c|c|c|c|}
\hline \multirow{3}{*}{$\begin{array}{l}\text { Parameters } \\
\text { Age (continuous) }\end{array}$} & \multicolumn{4}{|c|}{ Univariate analysis } & \multicolumn{4}{|c|}{ Multivariate analysis } \\
\hline & \multirow{2}{*}{$\frac{p}{0.330}$} & \multirow{2}{*}{$\begin{array}{c}\text { HR } \\
1.008\end{array}$} & \multicolumn{2}{|c|}{$\begin{array}{c}\text { 95\% CI } \\
\text { (lower/upper) }\end{array}$} & \multirow[t]{2}{*}{$p$} & \multirow[t]{2}{*}{ HR } & \multicolumn{2}{|c|}{$\begin{array}{c}\text { 95\% CI } \\
\text { (lower/upper) }\end{array}$} \\
\hline & & & 0.992 & 1.023 & & & & \\
\hline Gender (female vs. male) & 0.670 & 0.939 & 0.702 & 1.256 & & & & \\
\hline Smoking history (2/3/4/5 vs. 1$)$ & 0.662 & 0.912 & 0.604 & 1.377 & & & & \\
\hline Clinical stage (III/IV vs. I/II) & $<0.001$ & 2.646 & 1.942 & 3.606 & 0.005 & 1.687 & 1.168 & 2.437 \\
\hline Nodal status (positive vs. negative) & $<0.001$ & 2.569 & 1.912 & 3.452 & $<0.001$ & 1.906 & 1.346 & 2.700 \\
\hline Residual tumors (yes vs. no) & $<0.001$ & 3.937 & 2.204 & 7.033 & 0.001 & 2.650 & 1.463 & 4.801 \\
\hline UBE2D1 RNA expression (continuous) & 0.007 & 1.469 & 1.113 & 1.939 & 0.029 & 1.359 & 1.031 & 1.791 \\
\hline
\end{tabular}

TABLE 3: Univariate and multivariate analyses of recurrence-free survival in patients with LUAD.

\begin{tabular}{|c|c|c|c|c|c|c|c|c|}
\hline \multirow{3}{*}{$\begin{array}{l}\text { Parameters } \\
\text { Age (continuous) }\end{array}$} & \multicolumn{4}{|c|}{ Univariate analysis } & \multicolumn{4}{|c|}{ Multivariate analysis } \\
\hline & \multirow{2}{*}{$\frac{p}{0.323}$} & \multirow{2}{*}{$\begin{array}{c}\mathrm{HR} \\
1.008\end{array}$} & \multicolumn{2}{|c|}{$\begin{array}{c}\text { 95\% CI } \\
\text { (lower/upper) }\end{array}$} & \multirow[t]{2}{*}{$p$} & \multirow[t]{2}{*}{ HR } & \multicolumn{2}{|c|}{$\begin{array}{c}\text { 95\% CI } \\
\text { (lower/upper) }\end{array}$} \\
\hline & & & 0.992 & 1.025 & & & & \\
\hline Gender (female vs. male) & 0.574 & 1.097 & 0.794 & 1.516 & & & & \\
\hline Smoking history (2/3/4/5 vs. 1$)$ & 0.435 & 1.208 & 0.752 & 1.939 & & & & \\
\hline Clinical stage (III/IV vs. I/II) & 0.006 & 1.711 & 1.168 & 2.506 & 0.285 & 1.288 & 0.810 & 2.048 \\
\hline Nodal status (positive vs. negative) & 0.003 & 1.633 & 1.178 & 2.264 & 0.183 & 1.308 & 0.881 & 1.942 \\
\hline Residual tumors (yes vs. no) & $<0.001$ & 3.808 & 1.838 & 7.892 & 0.008 & 2.743 & 1.297 & 5.803 \\
\hline UBE2D1 RNA expression (continuous) & $<0.001$ & 1.958 & 1.443 & 2.657 & $<0.001$ & 1.842 & 1.353 & 2.508 \\
\hline
\end{tabular}




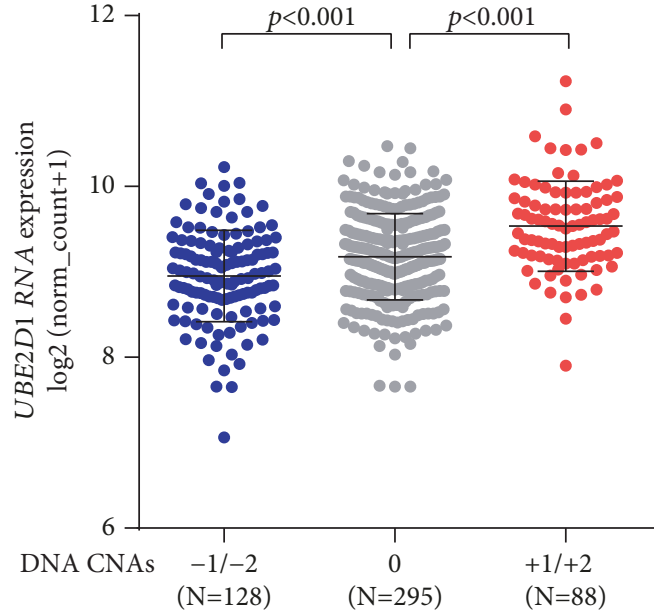

Figure 5: The association between UBE2D1 CNAs and UBE2D1 RNA expression. Comparison of UBE2D1 RNA expression in different CNA groups. CNAs were defined as homozygous deletion $(-2)$, heterozygous loss $(-1)$, copy neutral (0), low-level copy gain $(+1)$, and high-level amplification $(+2)$.

crucial role in human carcinogenesis, including NSCLC. P53 inactivation is closely associated with lung adenocarcinoma initiation, progression, and metastasis via multiple signaling pathways [16]. In Kras-driven LUAD, Notch1 initiates carcinogenesis by suppressing p53-mediated apoptosis through the regulation of p53 stability [17]. P53 inactivation can lead to DDX3 loss, which subsequently results in Slug-suppressed E-cadherin expression via decreased MDM2-mediated Slug degradation [18]. The NSCLC patients with $p 53$ inactivation also have poor survival outcomes [18]. P53 degradation directly lowers the p53-dependent transcription of the tumor suppressors RAD51 and $p 21$ and the upregulation of the oncogene SOX2 in LUAD [19]. A recent study showed that the UBE2Ds, together with RNF138, accumulate at damaged-DNA sites and promote DNA repair via promoting CtIP ubiquitylation and accrual [20]. In fact, the therapeutic effect of the current chemo- and radiotherapies mainly relies on inducing DNA damage of cancer cells. Therefore, upregulation of UBE2Ds in cancer cells may result in a weakened effect of the DNA-damaging anticancer therapy and rapid recovery after the damage. The key molecules in the DNA damage response (DDR) pathways have also been considered ideal targets for therapeutic intervention, including the E2s [21]. These mechanisms help explain why UBE2D1 upregulation is associated with poor OS and RFS in LUAD patients.

Although we confirmed the potential prognostic value of UBE2D1 RNA expression in LUAD, the mechanisms underlying its dysregulation have not been explored. In this study, we examined the association between UBE2D1 RNA expression and the CNAs of its DNA, and the results showed that DNA amplification was common in LUAD patients $(88 / 551,16.0 \%)$ and was associated with significantly upregulated UBE2D1 RNA expression, compared to the copy neutral patients. These findings suggest that DNA amplification might be an essential cause of upregulated UBE2D1 in LUAD.

\section{Conclusion}

UBE2D1 RNA expression might only serve as an independent prognostic indicator of unfavorable OS and RFS in LUAD, but not in LUSC. DNA amplification might be an essential cause of upregulated UBE2D1 RNA expression in LUAD.

\section{Data Availability}

All TCGA data used in this study can be obtained via the UCSC Xena browser (https://xenabrowser.net/).

\section{Conflicts of Interest}

The authors have no conflict of interest.

\section{References}

[1] Y. H. Jiang and A. L. Beaudet, "Human disorders of ubiquitination and proteasomal degradation," Current Opinion in Pediatrics, vol. 16, no. 4, pp. 419-426, 2004.

[2] C. Chen and L. E. Matesic, "The Nedd4-like family of E3 ubiquitin ligases and cancer," Cancer and Metastasis Reviews, vol. 26, no. 3-4, pp. 587-604, 2007.

[3] M. C. Rodrigo-Brenni and D. O. Morgan, "Sequential E2s drive polyubiquitin chain assembly on APC targets," Cell, vol. 130, no. 1, pp. 127-139, 2007.

[4] M. Windheim, M. Peggie, and P. Cohen, "Two different classes of E2 ubiquitin-conjugating enzymes are required for the mono-ubiquitination of proteins and elongation by polyubiquitin chains with a specific topology," Biochemical Journal, vol. 409, no. 3, pp. 723-729, 2008.

[5] C. P. Grou, A. F. Carvalho, M. P. Pinto et al., "Members of the E2D (UbcH5) family mediate the ubiquitination of the conserved cysteine of Pex $5 p$, the peroxisomal import receptor," Journal of Biological Chemistry, vol. 283, no. 21, pp. 1419014197, 2008.

[6] P. S. Brzovic, A. Lissounov, D. E. Christensen, D. W. Hoyt, and R. E. Klevit, "A UbcH5/ubiquitin noncovalent complex is required for processive BRCA1-directed ubiquitination," Molecular Cell, vol. 21, no. 6, pp. 873-880, 2006.

[7] R. S. Ranaweera and X. Yang, "Auto-ubiquitination of Mdm2 enhances its substrate ubiquitin ligase activity," Journal of Biological Chemistry, vol. 288, no. 26, pp. 18939-18946, 2013.

[8] M. K. Saville, A. Sparks, D. P. Xirodimas et al., "Regulation of p53 by the ubiquitin-conjugating enzymes UbcH5B/C in vivo," Journal of Biological Chemistry, vol. 279, no. 40, pp. 42169-42181, 2004.

[9] M. Tokumoto, Y. Fujiwara, A. Shimada et al., "Cadmium toxicity is caused by accumulation of p53 through the downregulation of Ube $2 d$ family genes in vitro and in vivo," Journal of Toxicological Sciences, vol. 36, no. 2, pp. 191-200, 2011.

[10] X. X. Sun, K. B. Challagundla, and M. S. Dai, "Positive regulation of p53 stability and activity by the deubiquitinating enzyme otubain 1," The EMBO Journal, vol. 31, no. 3, pp. 576-592, 2012.

[11] E. Varfolomeev, T. Goncharov, A. V. Fedorova et al., "c-IAP1 and c-IAP2 are critical mediators of tumor necrosis factor $\alpha$ (TNF $\alpha$ )-induced NF- $\kappa$ B activation," Journal of Biological Chemistry, vol. 283, no. 36, pp. 24295-24299, 2008. 
[12] S. Shukla, U. S. Allam, A. Ahsan et al., "KRAS protein stability is regulated through SMURF2: UBCH5 complex-mediated $\beta$ TrCP1 degradation," Neoplasia, vol. 16, no. 2, pp. 115-1W5, 2014.

[13] "Interactive human protein atlas launches," Cancer Discovery, vol. 5 , no. 4 , p. 339, 2015.

[14] M. Uhlén, L. Fagerberg, B. M. Hallström et al., "Proteomics. Tissue-based map of the human proteome," Science, vol. 347, no. 6220, article 1260419, 2015.

[15] "Human protein atlas," http://www.proteinatlas.org.

[16] Y. H. Wu, T. C. Wu, J. W. Liao, K. T. Yeh, C. Y. Chen, and H. Lee, "p53 dysfunction by xeroderma pigmentosum group $\mathrm{C}$ defects enhance lung adenocarcinoma metastasis via increased MMP1 expression," Cancer Research, vol. 70, no. 24, pp. 10422-10432, 2010.

[17] S. Licciulli, J. L. Avila, L. Hanlon et al., "Notch1 is required for Kras-induced lung adenocarcinoma and controls tumor cell survival via p53," Cancer Research, vol. 73, no. 19, pp. 59745984, 2013.

[18] D. W. Wu, M. C. Lee, J. Wang, C. Y. Chen, Y. W. Cheng, and H. Lee, "DDX3 loss by p53 inactivation promotes tumor malignancy via the MDM2/Slug/E-cadherin pathway and poor patient outcome in non-small-cell lung cancer," Oncogene, vol. 33, no. 12, pp. 1515-1526, 2013.

[19] K. Chen, K. Wu, S. Cai et al., "Dachshund binds p53 to block the growth of lung adenocarcinoma cells," Cancer Research, vol. 73, no. 11, pp. 3262-3274, 2013.

[20] C. K. Schmidt, Y. Galanty, M. Sczaniecka-Clift et al., "Systematic E2 screening reveals a UBE2D-RNF138-CtIP axis promoting DNA repair," Nature Cell Biology, vol. 17, no. 11, pp. 1458-1470, 2015.

[21] N. J. Curtin, "DNA repair dysregulation from cancer driver to therapeutic target," Nature Reviews Cancer, vol. 12, no. 12, pp. 801-817, 2012. 


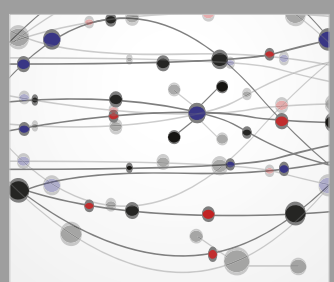

The Scientific World Journal
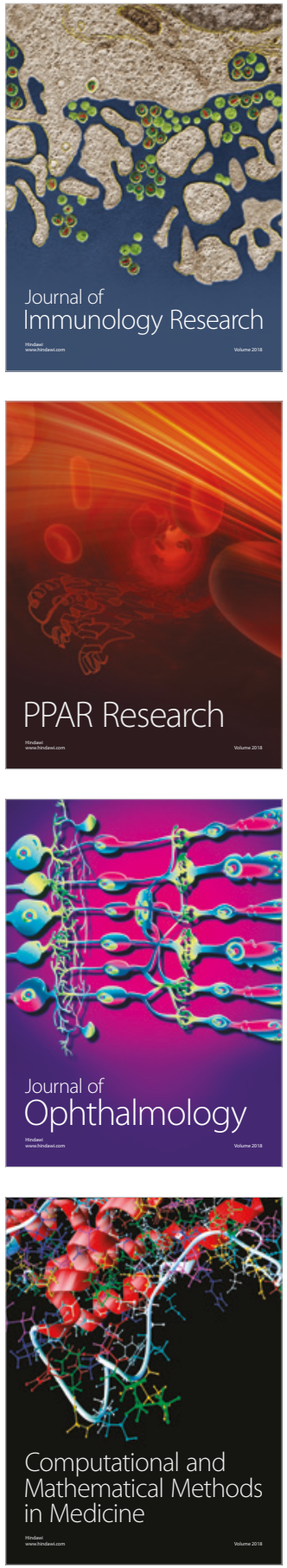

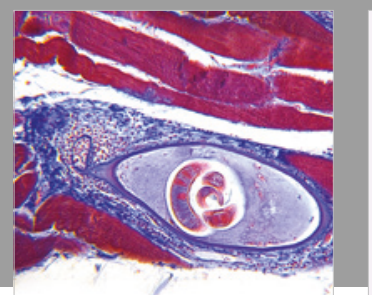

Gastroenterology Research and Practice

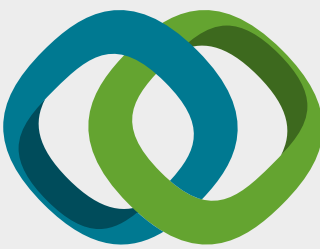

\section{Hindawi}

Submit your manuscripts at

www.hindawi.com
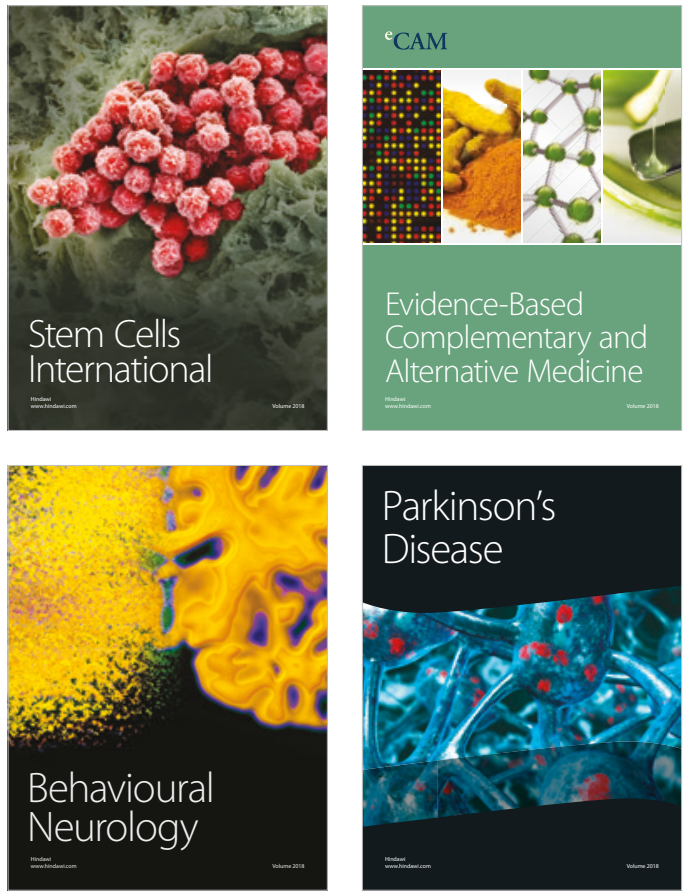

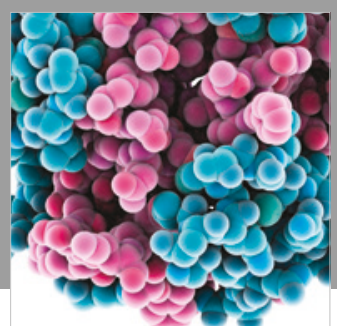

ournal of

Diabetes Research

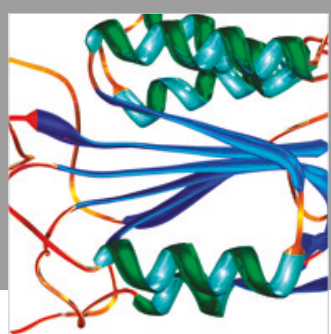

Disease Markers
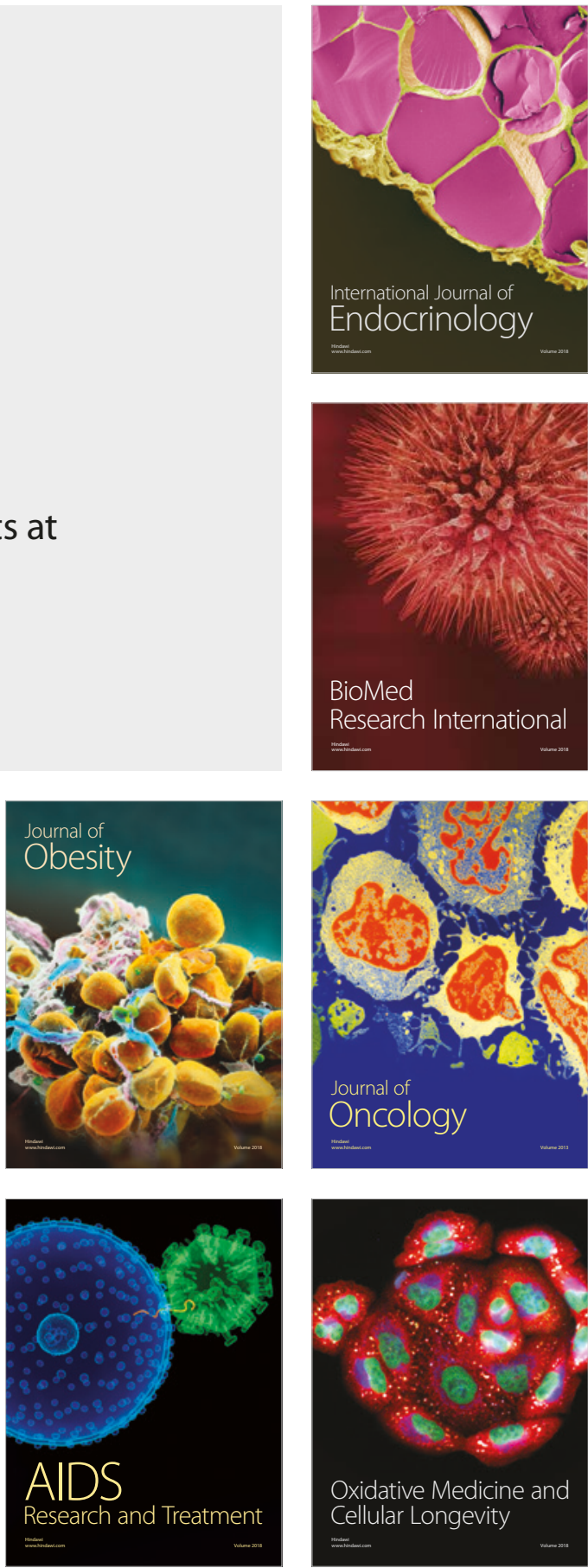